ISSN: 1130-2887 - eISSN: 2340-4396

DOI: https://doi.org/10.14201/alh.21462

\title{
LA CALIDAD DE LA RESPONSIVIDAD DE GOBIERNOS DE IZQUIERDA EN PAÍSES DE AMÉRICA LATINA: LA PERCEPCIÓN DE LA CIUDADANÍA
}

\author{
The quality of responsiveness of leftist governments in Latin American countries: \\ perception of the citizenry
}

\author{
Maria do Socorro Sousa BRAGA 드 \\ Universidade Federal de São Carlos \\ $\triangle$ msbraga@ufscar.br
}

\author{
Kátia Alves FUKUSHIMA \\ Universidade Federal do Espírito Santo \\ \katia.fukushima@ufes.br
}

Envío: 2019-09-21

Aceptado: 2020-08-23

First View: 2020-09-28

Publicación: 2020-12-22

RESUMEN: El objetivo de este trabajo es analizar el grado de responsividad, privilegiando la percepción de los ciudadanos sobre los gobiernos de la «izquierda moderada» de Brasil (2002-2010) y de Chile (2006-2010) y de la «izquierda contestataria» en Bolivia (2006-2014) y en Venezuela (1999-2013). Para ello se crearon indicadores de responsividad. Los resultados encontrados indican que hay una importante variación en el grado de responsividad de esas democracias gobernadas por las diferentes izquierdas.

Palabras clave: gobiernos de izquierda; responsividad; América Latina; izquierda moderada; izquierda contestataria.

ABSTRACT: The objective of this paper is to analyze the degree of responsiveness, privileging the perception of citizens about the «moderate left» governments of Brazil (2002-2010) and Chile (20062010) and the «contestatory left» in Bolivia (2006-2014) and Venezuela (1999-2013). To this end, responsivity indicators were created. The results indicate that there is an important difference in the degree of responsiveness in those democracies governed by the different lefts.

Keywords: left governments; responsiveness; Latin America; moderate left; contestatory left. 


\section{INTRODUCCIÓN ${ }^{1}$}

A pesar de los avances recientes de amplia bibliografía sobre la llegada de partidos de izquierda en los gobiernos de diversos países de América Latina en la primera década del siglo XXI, los análisis todavía son incipientes y carecen de un enfoque más estructurado y que sea capaz de evaluar los desdoblamientos de esos regímenes democráticos que contaron con gobiernos administrados por estas fuerzas en los últimos años. Este artículo, por lo tanto, fruto de dos investigaciones académicas, busca unir dos crecientes literaturas sobre los procesos políticos latinoamericanos. La primera de ellas, sobre las fuerzas de izquierda de la región que ascendieron al poder entre el final del siglo XX y principios del siglo XXI. La segunda, sobre el desempeño y la calidad de la democracia en América Latina tras el largo período de redemocratización en la mayoría de los casos analizados. Con el avance en el proceso de consolidación democrática en la región, los trabajos sobre la transición de los regímenes autoritarios fueron siendo gradualmente reemplazados por análisis sobre los avances y obstáculos de los regímenes democráticos latinoamericanos. Trabajos como el de Domínguez y Shifter (2008), Hagopian y Mainwaring (2005) y Mainwaring y Scully (2010) reunieron ideas de diferentes investigadores que buscaron desvelar los caminos recorridos por los regímenes democráticos latinoamericanos en las últimas décadas, además de evaluar el desempeño de políticas públicas en una serie de áreas.

Aunque se inserta en un enfoque más amplio de la evaluación sobre los regímenes democráticos en América Latina, este artículo está más cerca de los trabajos que buscaron analizar, en perspectiva comparada, la calidad de la democracia. Los trabajos como los de Altman y Pérez-Liñan (2002), Hagopian (2005) y Levine y Molina (2007, 2011) abrieron interesantes perspectivas de análisis sobre el tema al definir claramente las dimensiones que deben ser observadas y sugirieron indicadores empíricos capaces de medir cambios en esas dimensiones, posibilitando así una comparación diacrónica entre los países. Sin embargo, ninguno de estos autores se preocupó exclusivamente por los desdoblamientos de los gobiernos de izquierda de la región sobre la calidad de la democracia en sus países.

La justificación para dar cuenta de esa laguna en la literatura está relacionada con la importancia simbólica y programática de la llegada de esas fuerzas de izquierda a los gobiernos de esas nuevas democracias, consideradas como pertenecientes a la tercera ola de democratización. Hace ya un tiempo, al margen de las esferas decisivas de esas naciones, estos grupos se constituyeron en portavoces de amplios sectores de las poblaciones latinoamericanas excluidos política, social y económicamente, redundando en la ruptura con el patrón histórico de circulación de las élites nacionales. Además, el ascenso de esos grupos marcó de forma simbólica la aproximación de la población a la esfera de la política. Agregadores de programas políticos alternativos a las fuerzas de centro y de derecha, hasta entonces prevalecientes

1. Este artículo es parte del proyecto de investigación Evaluando la calidad de la democracia en los países de América Latina (II), apoyado con una beca de productividad del Consejo Nacional de Desarrollo Científico y Tecnológico (CNPq). Agradecemos a los revisores externos, así como a los editores de América Latina Hoy por los comentarios y sugerencias que contribuyeron en gran medida a mejorar este artículo. Las limitaciones que aún quedan son de nuestra exclusiva responsabilidad. 
en la región, esos proyectos fueron responsables, al menos en parte, de la elección de esas fuerzas para el Ejecutivo nacional. De ahí la relevancia de este artículo para evaluar comparativamente los resultados alcanzados por esos gobiernos en lo que se refiere a la calidad de la democracia, pero enfocándose, más específicamente, en una de sus dimensiones: la responsividad (responsiveness).

El objetivo de este trabajo es analizar el grado de responsividad, privilegiando la percepción de los ciudadanos sobre los gobiernos de izquierda «moderada» de Brasil con el gobierno de Lula (2002-2010) y de Chile con el gobierno de Bachelet (2006-2010), en comparación con la izquierda «contestataria» en Bolivia con el gobierno de Evo Morales ${ }^{2}$ (2006-2014) y en Venezuela con el gobierno de Hugo Chávez (1999-2013) (Madrid, Hunter y Weyland, 2010; Madrid, 2008 y Weyland, 2009).

Para dar cuenta de ese objetivo organizamos este artículo de la siguiente manera: en la próxima sección discutimos la relación entre democracia y responsividad y los principales conceptos que orientan este análisis. En la tercera sección, describimos cuáles son los principales mecanismos institucionales que afectan el proceso de representación y responsividad en los sistemas políticos comparados. Después, observamos los indicadores creados sobre la percepción del elector, bajo administraciones de los gobiernos de izquierda en el período de 1996 a 2014, con respecto al régimen democrático, al grado de efectividad de los gobiernos, a la confianza en los partidos políticos y en el Congreso Nacional, así como a la importancia del voto. Por último, analizamos las variaciones en las percepciones de los electores que viven bajo las diferentes administraciones ejecutando regresiones del tipo de paneles con errores robustos corregidos. En la última sección hacemos las consideraciones finales.

\section{DEMOCRACIA Y RESPONSIVIDAD: MARCO CONCEPTUAL}

¿Cuáles son los efectos de los gobiernos de izquierda sobre la calidad de la democracia en América Latina? Para pensar en esta cuestión vamos a presentar en esta sección las definiciones centrales que mueven este análisis: gobiernos de izquierda, calidad de la democracia y responsividad.

Entre los trabajos clásicos, como el de Bobbio (1995), definimos, en términos ideológicos, como gobiernos de izquierda aquellos que tienen, como una de sus prioridades, la búsqueda de una mayor igualdad tanto en el aspecto socioeconómico, con la reducción de la disparidad entre las clases sociales, como en el status de diferentes grupos, defendiendo, por ejemplo, más equidad entre géneros y etnias. En vista de la definición de la izquierda, podemos distinguirla de la derecha por el valor dado a la igualdad. Mientras que para la primera la igualdad es un ideal, para la segunda, no lo es. La derecha se detiene en la «igualdad frente a la ley, que implica únicamente el deber por parte del juez de aplicar las leyes de una manera imparcial y en la igual libertad», que caracteriza lo que Bobbio llamó «igualitarismo mínimo»

2. Este artículo analizó los dos primeros mandatos de Evo Morales. Sin embargo, el gobernó Bolivia hasta 2019, cuando renunció a la presidencia después de una serie de manifestaciones violentas y la presión de las Fuerzas Armadas. 
(Bobbio, 2011, p. 135). La izquierda critica la idea de la virtud salvacionista en el mercado, mientras que la derecha la promueve, como podemos ver en su defensa de la política económica neoliberal ${ }^{3}$. Tanto ideológicamente como en acción política, aunque en diferentes grados, podemos clasificar a los gobiernos analizados aquí como gobiernos de izquierda. Sin embargo, es importante señalar que no existe un gobierno puramente izquierdista. Por el contrario, muchos gobiernos de izquierda pueden ser bastante ortodoxos en sus políticas, porque un gobierno está compuesto por varios poderes integrados por diferentes actores que no están necesariamente vinculados a la izquierda.

En este marco teórico, Madrid, Hunter y Weyland (2010), Madrid (2008) y Weyland (2009) apuntan, a partir de las especificidades de los países gobernados por las izquierdas en América Latina, la existencia de tipos distintos de izquierdas. En ese sentido, los gobiernos seleccionados como estudio de caso en este artículo son clasificados por los autores como izquierdas «contestatarias» (en el caso de los gobiernos de Hugo Chávez en Venezuela y de Evo Morales en Bolivia) y como izquierdas «moderadas» (en el caso de los gobiernos de Lula en Brasil y de Bachelet en Chile).

En cuanto a la definición del régimen democrático, seguimos los requisitos establecidos por Diamond y Morlino (2005), que, a su vez, derivan del clásico trabajo de Dahl (1971). Según los autores, una democracia requiere: a) sufragio universal; b) elecciones libres, frecuentes, justas y competitivas; c) más de un partido político efectivo; y d) fuentes de información alternativas. En el marco de los cuatro requisitos anteriores, los autores aclaran también que las democracias no deben estar sometidas a las restricciones por parte de grupos e instituciones -internas o externas-, sino que rindan cuentas directa o indirectamente de sus acciones a la población y que para haber elecciones libres y justas es necesario que los regímenes cuenten con libertades civiles y políticas que permitan a los ciudadanos organizarse en torno a sus intereses y posiciones políticas (pp. x-xi).

De acuerdo con Diamond y Morlino (2005), es posible definir la calidad de la democracia a partir de tres elementos: (a) libertad, (b) igualdad política y (c) control popular sobre políticas públicas y políticos de forma institucionalizada. Para los autores, una democracia de calidad es aquella en que el régimen cuenta con amplia legitimidad y en la que los ciudadanos, individualmente o asociados, encuentran extensa libertad e igualdad política y poseen el poder de evaluar y controlar si el gobierno cumple con sus funciones de acuerdo con la ley (p. xii). Más detalladamente, los autores proponen un camino para la evaluación de la calidad de las democracias en función de los procedimientos, del contenido y de los resultados de los regímenes democráticos. Siguiendo a Diamond y Morlino (2005), cinco dimensiones son procedimentales: existencia de un Estado de derecho, participación política, competencia política y accountability horizontal y vertical. Dos dimensiones se refieren a las cuestiones sustantivas: el respeto a las libertades civiles y políticas y la aplicación de igualdad política, social y económica. Por último, los autores se refieren a la dimensión de la responsividad, responsable de la vinculación de las dimensiones procedimentales y sustantivas al enfocarse en los resultados del sistema político, o sea, en las políticas públicas y leyes y cómo reflejan los intereses y demandas de los ciudadanos.

3. Esto, por supuesto, no significa que los gobiernos de izquierda no adopten políticas neoliberales. 
En este artículo estamos priorizando el análisis de esta última dimensión: la responsividad, que será entendida como el grado de capacidad del régimen de responder a las expectativas, intereses y demandas de los ciudadanos por medio de arreglos institucionalizados. En palabras de Powell (2005, p. 91), la responsividad «es lo que ocurre cuando el proceso democrático induce al gobierno a formular y aplicar las políticas que los ciudadanos desean. Cuando el proceso induce a estas políticas de forma consistente, podemos considerar una democracia de alta calidad».

De acuerdo con Powell (2005), el proceso que haría el sistema político responsivo ocurriría en etapas sucesivas. Estas etapas, a su vez, estarían vinculadas por estructuras de conexión existentes en el arreglo institucional del sistema político, cruciales para fomentar incentivos para el buen funcionamiento de cada uno de esos momentos y del proceso como un todo. La primera etapa -las preferencias estructurantes- representa la conexión de las preferencias y demandas de los votantes con las alternativas políticas relevantes del sistema representativo, que a su vez se definen en gran medida por los partidos políticos. En la segunda etapa -la agregación institucional- las preferencias electorales serían agregadas y transformadas en escaños parlamentarios, o sea, por medio de mecanismos institucionales los votos de los electores ayudarían a formar gobiernos. Y las instituciones discutidas son los tipos de sistema electoral y de sistema de gobierno. Por último, en la tercera y última etapa, la producción de políticas, los representantes traducirían las demandas sociales en decisiones políticas, resultando en políticas públicas y leyes, es decir, vincularía las preferencias de los electores a las decisiones de los políticos electos, transformadas en políticas públicas y su implementación. En ese caso, para acceder a la calidad de la responsividad del gobierno, debemos analizar cómo los electores evalúan las políticas públicas elaboradas e implementadas.

Powell indica cuáles son las desviaciones más comunes en las estructuras de conexión entre cada etapa, que pueden originar fallas y rupturas en el proceso de responsividad. En la primera etapa, cuando ocurre la conexión entre las preferencias individuales y las opciones políticas que dan lugar a preferencias colectivas estructurantes, las desviaciones pueden ser de tres tipos. En primer lugar, puede ocurrir un fuerte control de información política por parte del gobierno, dificultando el acceso del elector a las mismas. En ese contexto la transparencia y la claridad de las atribuciones de responsividad acaban siendo afectadas. Segundo, las desviaciones están relacionadas con las limitaciones de alternativas políticas presentadas a los electores. En ese caso el sistema de partidos no está abierto lo suficiente para que abarque puntos de vista diversos, consecuentemente puede haber sectores relevantes en la sociedad que no están contemplados en ese sistema político, prefiriendo abstenerse. Por último, el tercer problema está en la incoherencia de los partidos al presentar sus propuestas, afectando la inteligibilidad del elector al buscar distinguir las posiciones partidistas, dificultando así las preferencias electorales. La ausencia de claridad programática entre los partidos puede ser resuelta con un sistema de partidos conformado por partidos estables defensores de distintos proyectos para el país y que participan regularmente de las competiciones políticas ofreciendo al electorado un discurso nacional consistente con su programa. Además, la presencia de medios de comunicación de masas activos e independientes, de ciudadanos educados y de partidos vinculados al discurso a escala nacional «promueven un público informado cuyas preferencias son suficientemente coherentes para que la capacidad de respuesta sea significativa» (Powell, 2005, p. 100). 
Las desviaciones en la segunda etapa, la agregación institucional, están relacionadas con los condicionamientos y distorsiones de los sistemas electorales y de gobierno en la formación de gobiernos y en los atributos de sus gobernantes. Powell llama la atención sobre problemas como los de la sobre- o subrepresentación de las fórmulas que transforman votos en escaños, de la migración de los representantes electos entre los partidos, del uso de decretosley por el Ejecutivo, entre otros dispositivos existentes en cada tipo de diseño institucional adoptado por las democracias y que afectan la calidad de la responsividad de los gobiernos. Para dar cuenta de estos posibles obstáculos el autor sugiere la adopción de parlamentos proporcionales por sus virtudes inclusivas, representando la diversidad de opiniones de la sociedad y la formación de partidos coherentes en su programa y consistentes en su discurso nacional cuyos gobernantes deberían ser responsables de formular e implementar políticas de inclusión social.

Por último, las desviaciones en la etapa de la producción de políticas pueden incluir: i) problemas de corrupción, ya que las desviaciones de recursos públicos para otros fines restringen el monto de dinero para que el gobierno efectúe políticas públicas; ii) influencia de grupos de poder, por medio de lobbies, grupos económicos que buscan ser beneficiados en detrimento de la mayoría de la población; y iii) baja cualificación técnica de la burocracia, que puede impedir que las decisiones políticas alcancen a los beneficiarios. Para resolver estos problemas o, antes, impedir que esos obstáculos dificulten la responsividad del proceso decisorio, el autor resalta las siguientes condiciones: en el caso de la corrupción, es fundamental que los partidos en el gobierno rindan cuentas de los gastos regularmente, viabilizando la transparencia de las cuentas públicas. En relación con el segundo problema, debe haber rendición de cuentas horizontal, es decir, entre los poderes que conforman el sistema político. Finalmente, en lo que se refiere a la incompetencia del cuerpo burocrático, el Estado tendría que crear mecanismos de selección por la meritocracia para tener funcionarios entrenados, con capacidad burocrática ${ }^{4}$.

Como hemos discutido hasta ahora, el modelo de responsividad de Powell (2004) nos plantea ante diversos desafíos para evaluar y comparar la calidad de la responsividad de los gobiernos de izquierda. Como vimos, el impacto del arreglo institucional en el comportamiento de los actores políticos tiene un papel central en el modelo teórico de Powell. Por eso, el primer momento del análisis de la calidad de la responsividad es la identificación de esas instituciones vigentes en cada país, así como de sus efectos e incentivos para el buen funcionamiento de las diferentes etapas del proceso de representación.

En este artículo vamos a identificar y describir las principales instituciones políticas que están relacionadas con la responsividad en los países estudiados y que afectan, por lo tanto, al comportamiento y la percepción de representados y representantes. Y también analizaremos la calidad de la responsividad desde el punto de vista de los ciudadanos, centrándonos en sus percepciones y preferencias políticas.

4. Es decir, servidores públicos autónomos, bien organizados y calificados y que sean capaces de implementar políticas de manera efectiva y con un mínimo de corrupción. 


\section{LA CALIDAD DE LA RESPONSIVIDAD DE GOBIERNOS EN PAÍSES DE AMÉRICA LATINA: LA PERCEPCIÓN DE LA CIUDADANÍA}

\section{III.1. Los contextos institucionales y políticos}

El inicio del siglo XXI fue marcado en América Latina por el ascenso de gobiernos de izquierdas, a ejemplo de Chávez en Venezuela en 1999; de Lula en Brasil en 2003; de Evo Morales en Bolivia, y de Michelle Bachelet en Chile en 2006. Esta ola se inserta en el contexto de crisis económicas, políticas y sociales por las que la región pasaba a finales de la década de 1990. La solución por parte de la mayoría de los gobiernos latinoamericanos fue la adopción de políticas económicas neoliberales 5 . Sin embargo, vale la pena mencionar que la adopción de políticas neoliberales en los países latinoamericanos no puede entenderse simplemente como una imposición externa, estas políticas también han sido resultado de decisiones nacionales adoptadas desde mediados de los años ochenta por regímenes políticos democráticos (Ocampo y Ros, 2011).

Según Weyland (2000, p. 79), los gobiernos latinoamericanos «no fueron prudentes al elegir programas de choque». La consecuencia para los latinoamericanos fue el estancamiento y/o recesión (como en la «década perdida» de 1980) o el pico de crecimiento seguido por el colapso económico (como en Argentina) (Harvey, 2007, p. 163). Además, someter a los países latinoamericanos a las fuerzas del mercado no resolvió el problema de la deuda externa, que tanto preocupaba a los organismos internacionales (a ejemplo del FMI).

El contexto demostraba que no se trataba solo de una crisis económica, sino también de una crisis política y social expresada en una serie de protestas contra los gobiernos hasta entonces vigentes, ante el empeoramiento en los índices sociales y, consecuentemente, la convulsión social frente al aumento de las deudas públicas (interna y externa). Sin negar las singularidades de cada país, podemos afirmar que la crisis económica y, por tanto, la desconfianza de la población latinoamericana en las instituciones políticas, demostrada en las manifestaciones sociales (contra la corrupción y la desigualdad) y en el «voto castigo» constituyeron la brecha para los candidatos de la izquierda en América Latina 6 .

La presencia de esas fuerzas en el Poder Ejecutivo representaba la alternativa a los gobiernos neoliberales y la posibilidad de gobiernos más inclusivos. Sin embargo, cada uno de esos gobernantes «a la izquierda» presentó un camino sui generis, a medida que se enfrentaban a circunstancias históricas, políticas, económicas, sociales y culturales específicas de sus

5. Entre las políticas neoliberales están: la disciplina fiscal; la reforma tributaria; tasas de cambio de acuerdo con las leyes de mercado; la liberalización del comercio; el fin de las restricciones a las inversiones extranjeras; las privatizaciones de empresas estatales y los cambios de prioridades en el gasto público. Aunque las ideas neoliberales habían sido presentadas previamente por institutos de economía, agencias internacionales y el gobierno de los Estados Unidos, el conocido Consenso de Washington, según Batista (1994, p. 99), ratificó «la propuesta neoliberal que el gobierno estadounidense había recomendado insistentemente, a través de las entidades antes mencionadas, como una condición para otorgar cooperación financiera externa, bilateral o multilateral».

6. No fue en todos los países, sin embargo, que las izquierdas obtuvieron éxitos electorales tras la crisis de los años 1990. 
países. Como ya mencionamos, Madrid, Hunter y Weyland (2010), Madrid (2008) y Weyland (2009) apuntaron a la presencia de diferentes izquierdas: la «izquierda contestataria» (Venezuela y Bolivia), la cual persigue sus metas desafiando el neoliberalismo y las restricciones de la globalización; y la «izquierda moderada» (Brasil y Chile), que actúa respetando las limitaciones económicas. En el análisis de los autores, los partidos de izquierda de Brasil (Partido de los Trabajadores - PT) y de Chile (Partido Socialista - PS) se trasladaron al centro, mientras que en Bolivia (Movimiento al Socialismo - MAS) y Venezuela (Partido Socialista Unido de Venezuela - PSUV) se radicalizaron oponiéndose al modelo liberal.

Roberts (2008, p. 87) acerca a los partidos de izquierda chileno (PS) y brasileño (PT) a la izquierda socialdemócrata europea, «una alternativa que combina la democracia representativa con una economía de mercado e iniciativas del Estado para reducir las desigualdades y promover la ciudadanía social». Esto porque se mueven en contextos de pluralismo institucionalizado, con mecanismos de control que ponen límites a sus ambiciones reformistas. La «izquierda contestataria» rompe con los acuerdos graduales cuidadosamente negociados y apunta a «ambiciosos proyectos transformadores y nuevas formas de soberanía popular».

En vista del contexto, es fundamental discutir las diferencias entre estos dos tipos de gobiernos en lo que se refiere a las configuraciones político-institucionales que operaron el proceso político-electoral, pues, como vimos en la sección anterior, la calidad de la responsividad de una democracia, según Powell (2005), depende de las características institucionales del proceso de representación política.

Con respecto a las Constituciones de estos países, la brasileña data de 1988 en el contexto de la redemocratización, que incluye una serie de innovaciones sobre el Estado de derecho y los mecanismos de la democracia directa, tales como plebiscito, expansión del sufragio e iniciativas populares en diferentes áreas de políticas públicas. La Constitución chilena ${ }^{7}$ (1980) tiene un carácter peculiar porque fue promulgada durante un régimen militar. Dicha Carta Magna es extremadamente restrictiva en términos de mecanismos de participación. Tanto en Venezuela como en Bolivia, la Constitución fue promulgada, mediante referéndum popular, por los gobiernos de Chávez (en 1999) y de Evo Morales (en 2009), respectivamente. Tales documentos presentaron cambios más profundos, marcando un proceso de rupturas con el statu quo hasta entonces vigente y la refundación del Estado. Entre los elementos más importantes de la nueva Constitución venezolana está la ampliación de mecanismos participativos y de control social (artículo 184), como la utilización de plebiscitos y referendos; la posibilidad de convocar referendo revocatorio para todos los cargos y magistraturas de elección popular - recall (artículo 72); y la garantía de que los pueblos indígenas podrán elegir a tres diputados (artículo 186) (Venezuela en 1999). A partir de esos elementos, Pogrebinschi (2013, p. 8) afirma que la participación aparece en el gobierno de Chávez como «un medio para que la representación sea más legítima, transparente, receptiva y, por lo tanto, más democrática». En la misma línea, siguió la Constitución de Bolivia, con la ampliación de los derechos indígenas y la creación de circunscripciones de pueblos originarios campesinos; la sustitución del

7. El presidente de Chile, Sebastián Piñera, firmó el 23/12/2019 la ley que permite convocar a un plebiscito para cambiar la Constitución heredada de la dictadura de Pinochet (1973-1990). La votación está programada para el 25/10/2020 (https://www.servel.cl/). 
Congreso Nacional por la Asamblea Legislativa Plurinacional; la Asamblea y los Consejos pasan a tener carácter deliberativo y se refuerza el papel del Estado en la economía (Progrebinschi, 2013).

La presencia de mecanismos de participación contribuye a la calidad de la responsividad, ya que les permite a los ciudadanos participar en los asuntos públicos y garantizar su influencia directa en la decisión de las políticas públicas y en el control de los funcionarios del gobierno, haciéndolos responsables de sus acciones (Diamond y Morlino, 2005).

El contexto de un sistema de partidos con un mayor grado de institucionalización presupone que existe fundamentalmente la aceptación (y la movilización efectiva) de la política partidaria como el canal principal para procesar los conflictos y, en particular, la predisposición a considerar los resultados producidos por dinámica electoral, cualesquiera que sean. En contraste, los contextos de crisis del sistema partidario o sistemas de partidos débilmente institucionalizados «se caracterizan por la menor regularidad en los modelos de competición partidista, el débil arraigamiento del partido en la sociedad, por la menor legitimidad otorgada a los partidos; y por las débiles organizaciones partidistas, a menudo dominadas por líderes personalistas» (Mainwaring y Torcal, 2005, pp. 146-147).

Como podemos observar en la Tabla I, la izquierda moderada gobernó en contextos relativamente institucionalizados mientras la izquierda contestataria se situó en contextos de desestructuración del sistema partidista y/o de deslegitimación de los actores políticos tradicionales. El nivel de institucionalización del sistema de partidos es en gran medida producto de la historia política de cada país y, por lo tanto, dependiendo del contexto, un sistema de partidos institucionalizado puede sufrir cierta «desinstitucionalización». Este es el caso de Venezuela, que, antes de la década de 1990, la democracia venezolana fue considerada una de las más estables de la región. «Sin embargo, el derrumbe de la legitimidad de los partidos políticos más grandes y los cambios del sistema electoral generaron una ola de volatilidad y el surgimiento de un número extraordinariamente elevado de partidos y movimientos nuevos» (Payne et al., 2006, p. 172). Asimismo, Bolivia también pasó por una crisis en el sistema de partidos con la deslegitimación de los partidos tradicionales y el surgimiento de nuevos actores vinculados a los movimientos sociales.

Brasil y Chile presentaron un sistema de partidos institucionalizado con relativa estabilidad para los partidos y las reglas del juego. Como señaló Powell, la presencia de partidos estables favorece la responsividad. Por lo tanto, a este respecto, los gobiernos de la izquierda moderada están por delante de la izquierda contestataria. Todavía, al considerar el contexto de cada país, podemos afirmar que, ante la crisis del sistema de partidos en Venezuela y Bolivia, el proceso de institucionalización requiere tiempo para proporcionar partidos estables. Chile, aunque presenta un sistema de partidos institucionalizado y estable, es uno de los casos, según Payne et al. (2006, p. 175-176), «con porcentajes menores de ciudadanía que se identifica con algún partido» esto porque, «más allá de la tendencia general de indiferencia hacia la política que parece afectar a toda América Latina, la competencia electoral que impone la existencia de dos amplias coaliciones, una de centro-izquierda y otra de centro-derecha, haya debilitado los vínculos de la ciudadanía con los partidos individuales». 
TABLA I. CARACTERÍSTICAS DE LOS PARTIDOS Y SUS LÍDERES

\begin{tabular}{l|l|l|l|l}
\hline \multicolumn{2}{l|}{} & CONTEXTO & $\begin{array}{l}\text { N. }{ }^{\circ} \text { EFECTIVO } \\
\text { DE PARTIDOS }\end{array}$ & $\begin{array}{c}\text { CONSTRUCCIÓN } \\
\text { DE MAYORÍAS }\end{array}$ \\
\hline \multirow{2}{*}{$\begin{array}{l}\text { Izquierda } \\
\text { moderada }\end{array}$} & Brasil & Institucionalizado & Alto & $\begin{array}{l}\text { Mayorías dependientes } \\
\text { de la coalición }\end{array}$ \\
\cline { 2 - 5 } & Chile & Institucionalizado & Alto & $\begin{array}{l}\text { Mayorías dependientes } \\
\text { de la coalición }\end{array}$ \\
\hline \multirow{2}{*}{$\begin{array}{l}\text { Izquierda } \\
\text { contestataria }\end{array}$} & Bolivia & $\begin{array}{l}\text { Crisis Sistema } \\
\text { Partidario }\end{array}$ & Bajo & Mayoría absoluta \\
\cline { 2 - 5 } & Venezuela & $\begin{array}{l}\text { Crisis Sistema } \\
\text { Partidario }\end{array}$ & Bajo & Mayoría absoluta \\
\hline
\end{tabular}

Fuente: Elaboración propia con base en Payne et al. (2006) y Alcántara Sáez (2009, 2008).

En cuanto al número efectivo de partidos con presencia legislativa, Alcántara Sáez (2009, p. 76) observó que, al final de 2008, el número efectivo de partidos en el Legislativo era muy distinto en América Latina. En los casos de los países gobernados por la izquierda contestataria el número era inferior a 3,25 -considerado índice bajo, «reflejando a su vez la reducción de la competencia política polarizada en torno al binomio oficialismo-oposición»-. Los países gobernados por la izquierda moderada (Brasil y Chile $)^{8}$ presentaban índices superiores a 5 , por lo tanto, un número muy alto, lo que proyecta un multipartidismo extremo.

En cuanto a la construcción de mayorías, el análisis de Alcántara Sáez (2008) apuntó que la izquierda contestataria con Chávez y Evo Morales tenía mayorías absolutas en 2008, lo que permitía la implementación de políticas públicas sin trabas. La izquierda moderada con Bachelet y Lula poseía mayorías como resultados de coaliciones, sean de carácter estable o coyuntural.

Otro punto destacado por Powell es la representatividad de los diferentes grupos en el sistema de partidos. La baja participación de grupos minoritarios (como mujeres y pueblos indígenas) en la política es un obstáculo para la calidad de la responsividad. Para resolver el problema de la subrepresentación de las mujeres en la política, algunos países han adoptado las cuotas de género. En el caso de Brasil, existe una ley de cuotas que establece un mínimo del 30\% y un máximo del $70 \%$ de las vacantes para candidatos de cada género en las listas electorales. Si bien la ley de cuotas es un avance para la expansión de la participación femenina en la política brasileña, el registro de candidatas se ha constituido en candidaturas de «relleno» que son lanzadas por los partidos solo para cumplir con el porcentaje requerido por la Justicia Electoral, pero no están garantizando la llegada efectiva de las mujeres a la

8. En Chile, aunque el número efectivo de partidos es alto, la existencia de una amplia coalición estable, donde el presidente es apoyado por un partido mayoritario, lo aproxima a los países con bajos números de partidos efectivos. 
elección popular. En su primer mandato, Bachelet no pudo implementar la ley de cuotas de género para garantizar el acceso y participación de las mujeres en los espacios de poder político9. Aunque al comienzo de su gobierno Bachelet buscó avanzar estableciendo la paridad entre hombres y mujeres en los ministerios, esta medida no fue institucionalizada. En Chile el porcentaje de mujeres en las legislaturas está muy por debajo del promedio de la región. En Venezuela, el artículo referido a las cotas en la Ley Orgánica del Sufragio y Participación Política de 1998, que establecía un mínimo del 30\% por cada sexo, fue declarado inconstitucional y derogado, aunque en procesos electorales posteriores el Consejo Nacional Electoral (CNE) aprobó resoluciones especiales exigiendo paridad en las nóminas de candidatos. En los casos en que un partido no puede garantizar la paridad, la proporción de sus candidatos de ambos sexos no puede ser inferior al $40 \%$ ni superior al $60 \%{ }^{10}$. En Bolivia se aplica la paridad y alternancia en las listas de candidatas y candidatos para todos los cargos de gobierno y de representación y la paridad tiene rango constitucional (Freidenberg y Caminotti, 2015).

Con respecto a la inclusión de los pueblos indígenas en la política, la izquierda «contestataria» en Bolivia y Venezuela incluyó, en las nuevas Constituciones, políticas significativas para la representatividad de estos grupos. La Constitución venezolana establece que «al cuerpo legislativo deben agregarse tres diputados que son elegidos en representación de las comunidades indígenas». Lo mismo sucede en Bolivia, que en la reforma del 2009 ha incluido las siete Circunscripciones Especiales Indígena, Originario, Campesinas (Freidenberg y Došek, 2016).

La Tabla II muestra los sistemas electorales que fueron prácticamente mantenidos por los gobiernos de izquierda en el siglo XXI. Bolivia y Venezuela presentan un sistema mixto (de corrección), combinando representación proporcional con lista cerrada y con voto mayoritario en distritos uninominales. Para Negretto (2009, p. 75), ese sistema puede alcanzar un balance eficiente entre el protagonismo de los partidos políticos y los candidatos individuales a medida que combina el voto partidista y el voto personalizado. Además, el sistema mixto puede mantener altos grados de consistencia política y de disciplina de voto entre sus legisladores, al tiempo que crea incentivos para intercambios más estrechos entre los votantes y una parte de los representantes, así como una mayor rendición de cuentas de estos últimos.

El sistema binominal chileno ${ }^{11}$ se sitúa como la base principal en la formación de un sistema de vetos que logró crear y sostener un impasse político. De acuerdo con Payne et al. (2006, p. 46), el resultado de ese sistema es una forma particular de sistema mayoritario que favorece a los partidos mayores y, en especial, al segundo partido (o coalición) más importante ${ }^{12}$.

9. En 2015, el Congreso aprobó un proyecto de ley sobre reforma constitucional que incluye una cuota de género (Ley 20.840, artículo 1. https://www.leychile.cl/Navegar?idNorma=1077039).

10. Artículo 3, resolución n. ${ }^{o}$ 150625-147 del Consejo Nacional Electoral. https://oig.cepal.org/sites/ default/files/2015_res_ven.pdf.

11. Los partidos o coaliciones de partidos presentan listas de candidatos en circunscripciones binominales. Los votantes votan por un candidato, pero el partido (o la coalición) acumula los votos (Payne et al., 2006).

12. El fin del sistema binomial chileno ocurrió en el segundo mandato de Bachelet (20142018), período no analizado en este artículo (Ley n. 20.840 del 05/05/2015: http://www.leychile.cl/ Navegar?idNorma $=1077039)$. 
La configuración institucional vigente en Chile y el equilibrio de fuerzas políticas que perpetúa a través de fórmulas, como un sistema electoral que sobrerrepresenta la coalición de partidos de derecha y centro derecha y un sistema de decisión legislativo que contempla supramayorías para las materias de gran relevancia, conformando así un sistema de vetos, es precisamente lo que en última instancia se ha convertido en los canales ordinarios incapaces de representar y procesar el tipo de intereses y cuestiones que agitan los sectores movilizados (Pinto y Flisfisch, 2011, p. 157).

TABLA II. SISTEMAS ELECTORALES DE LOS CASOS ANALIZADOS

\begin{tabular}{|c|c|c|c|c|}
\hline IZQUIERDAS & PAÍS & SISTEMA & $\begin{array}{l}\text { FÓRMULA } \\
\text { ELECTORAL }\end{array}$ & AÑO \\
\hline \multirow{2}{*}{$\begin{array}{l}\text { Izquierda } \\
\text { contestataria }\end{array}$} & Bolivia & $\begin{array}{l}\text { Mixto: distritos uninominales } \\
+ \text { representación proporcional } \\
\text { - lista cerrada }\end{array}$ & $\begin{array}{l}\text { Plurinominal: } \\
\text { D'Hondt/ } \\
\text { Uninominal: } \\
\text { mayoría simple }\end{array}$ & $1994 / 2010$ \\
\hline & Venezuela & $\begin{array}{l}\text { Mixto: distritos uninominales } \\
+ \text { representación proporcional } \\
\text { - lista cerrada }\end{array}$ & $\begin{array}{l}\text { D’Hondt/ } \\
\text { mayoría simple }\end{array}$ & $1989 / 2009$ \\
\hline \multirow{3}{*}{$\begin{array}{l}\text { Izquierda } \\
\text { moderada }\end{array}$} & \multirow[t]{2}{*}{ Brasil } & $\begin{array}{l}\text { Representación proporcional - } \\
\text { lista abierta }\end{array}$ & \multirow[t]{2}{*}{ Hare/D’Hondt } & \multirow[t]{2}{*}{$1946 / 1950$} \\
\hline & & Magnitudes grandes & & \\
\hline & Chile & Binominal - lista abierta & D'Hondt & 1989 \\
\hline
\end{tabular}

Fuente: Observatorio de Reformas Políticas en América Latina (1978-2019): https://reformaspoliticas.org/ investigacion/tablas-de-datos/temas/sistema-electoral/.

El sistema proporcional brasileño ha generado distorsiones en términos de fortalecimiento del partido y la representación. Como la Constitución establece un límite mínimo (8) y máximo (70) para los diputados federales, los estados con menos votantes como Acre, Rondônia y Amapá están sobrerrepresentados, mientras que los estados con un alto contingente electoral como São Paulo, Minas Gerais y Río de Janeiro están subrepresentados. Para Payne et al. (2006, p. 63), las circunscripciones son muy amplias para que el voto para un candidato permita crear un vínculo estrecho entre electores y representantes.

Los cuatros países utilizan la fórmula D'Hondt, «que es menos proporcional y favorece a los partidos más grandes» (Freidenberg y Došek, 2016, p. 45). El método de distribución de escaños practicado en Brasil combina el uso de la cuota Hare, conocida en la legislación electoral como cociente electoral, con la de un sistema de divisores para los escaños distribuidos en las sobras (D’Hondt) (Nicolau, 2004, pp. 45-46).

Tanto Brasil y Chile como Bolivia son bicamerales (Cámara y Senado). Venezuela presentó cambios con el ascenso de Chávez (1999) al sustituir el Congreso bicameral por una 
Asamblea unicameral. El problema de introducir Asambleas unicamerales en detrimento de Congresos bicamerales es que el sistema puede volverse menos representativo. Sin embargo, puede ser más efectivo y participativo (Payne et al., 2006).

De acuerdo con Powell (2005), entre las desviaciones en la etapa de la producción de políticas se encuentran los problemas de corrupción y la influencia de grupos de poder. Para impedir que esos obstáculos dificulten la responsividad del proceso decisorio, el autor resalta que es fundamental la transparencia de las cuentas de los partidos y entre los poderes que conforman el sistema político. Como afirman Pinto y Flisfisch (2011, p. 133), «A mayor transparencia financiera, mayor es la posibilidad de representación política, que a su vez deriva en la mayor legitimidad democrática representativa».

Con respecto a los partidos, hemos verificado algunas medidas de control y transparencia, buscando evitar la corrupción y la influencia de los grupos de interés. El gobierno venezolano aprobó la Ley para la Defensa de la Soberanía Política y la Autodeterminación Nacional, que prohíbe a los partidos políticos, entre otras organizaciones con objetivos políticos, recibir donaciones extranjeras. En el caso chileno, las contribuciones corporativas anónimas a un partido deben canalizarse a través del Servicio Electoral Chileno, que luego entrega contribuciones al partido sin revelar la fuente de sus fondos, para que las contribuciones puedan ser monitoreadas (Londoño y Zovatto, 2015, p. 187). En Chile y Brasil existen límites máximos para las donaciones relacionadas con las elecciones. Bolivia limita las contribuciones a los partidos políticos durante los períodos fuera de campaña. Tanto los partidos brasileños como los chilenos tienen fondos públicos. Durante el gobierno de Lula en Brasil, se aprobó en 2006 el final de la verticalización obligatoria de las coaliciones partidistas y de reglas más rígidas en lo que se refiere al financiamiento de las campañas electorales ${ }^{13}$. Estas modificaciones están estrictamente vinculadas a la opinión pública insatisfecha con las noticias de corrupción en el interior del gobierno y de los partidos. Los gobiernos de izquierda en Venezuela y Bolivia han eliminado la financiación pública. Brasil, Chile y Bolivia requieren informes periódicos sobre financiamiento y gasto de partidos en propaganda y campañas electorales. Según Londoño y Zovatto (2015), la mayoría de los países latinoamericanos requieren legalmente que se publique la información financiera de los partidos y candidatos. Bolivia y Venezuela son excepciones a esta regla. Sin embargo, en la práctica, a menudo existen otros obstáculos para acceder a esta información, o la información proporcionada es incompleta o incorrecta. En Chile, por ejemplo, el acceso a la información es difícil y la calidad de la información es a menudo deficiente (Londoño y Zovatto, 2015).

Otras medidas propuestas por los gobiernos de izquierda están vinculadas a la rendición de cuentas horizontal. En Brasil, el gobierno creó el Portal de Transparencia, en el que los ciudadanos pueden encontrar información sobre cómo se utiliza el dinero público

13. Entre las reglas están: la cancelación de registro de candidatura o casación de mandato en caso de uso comprobado de «Caja 2»; la divulgación de recaudación y gastos por parte de los candidatos durante la campaña; la prohibición de donaciones de campaña de entidades y organizaciones no gubernamentales que reciban recursos públicos, y organizaciones de la sociedad civil de interés público; y la prohibición de showmicios (es decir, de actos públicos donde un candidato a un cargo político expone sus ideas a los votantes, generalmente acompañado por artistas musicales para atraer a un público mayor) y de la distribución de regalos por los candidatos (Melo, 2006, p. 56). 
y sobre asuntos relacionados con la gestión pública. En Chile, también se aprobó la Ley de Transparencia y Acceso a la Información Pública (Ley 20.285/2008) y con ella se creó el Consejo de Transparencia con el objetivo de controlar el cumplimiento de las normas de publicidad y transparencia y garantizar el derecho de acceso a la información (Varas, 2012). Los ministerios chilenos ahora tienen un Sistema Integral de Atención Ciudadana, portales de información y cartas de derechos ciudadanos. En Venezuela, desde la Constitución de 1999, el ciudadano ha ganado espacio gubernamental con el Poder Ciudadano, cuya función es mediar ¿en? los mecanismos de control y rendición de cuentas. En Bolivia, se creó el Ministerio de Transparencia y Lucha contra la Corrupción, que «incorpora el control social, la transparencia y el acceso a la información, la imprescriptibilidad, la retroactividad de la ley penal anticorrupción, la rendición pública de cuentas, la participación social en las políticas anticorrupción, temas que son desarrollados en la presente Política» ${ }^{14}$.

A partir de la evaluación del sistema electoral y de los cambios introducidos en el mismo podemos afirmar, en lo que se refiere a los gobiernos de izquierdas, que la izquierda moderada - por gobernar en un escenario en que los partidos políticos se encontraban relativamente institucionalizados y, por lo tanto, promover cambios requeriría una correlación de fuerzas entre los mismos- optó por medidas puntuales, sin moverse en la estructura institucional. En Venezuela y Bolivia, los cambios estructurales, como el cambio de la propia Constitución, encontraron terreno propicio ante un sistema partidista desestructurado.

En vista de la configuración del contexto institucional y político de los casos analizados, ponderaremos a continuación los resultados obtenidos a partir de indicadores que buscan demostrar el grado de responsividad de los distintos gobiernos de izquierda, a partir de la percepción de los ciudadanos.

\section{III.2. Análisis de los resultados}

Para evaluar el grado de responsividad del régimen democrático que se viene desarrollando en los gobiernos de izquierda moderada, de Brasil (2002-2010) y de Chile (2006-2010); y de la izquierda contestataria, en Bolivia (2006-2014) y en Venezuela (1999-2013), privilegiamos la percepción de los ciudadanos y creamos una base de datos para medir diversos indicadores de responsividad. Conforme a la Tabla III, dos de ellos, satisfacción con la situación política del país y preferencia por la democracia, buscan examinar si el grado de adhesión a la democracia aumentó o disminuyó cuando los gobiernos de izquierda asumieron el control de esos regímenes. La confianza en los partidos políticos, en el voto y en el Congreso Nacional son indicadores más directos sobre la responsividad porque miden la eficacia de tres instituciones primordiales para la supervivencia de la democracia representativa al estructurar las elecciones colectivas nacionales y formar gobiernos. Finalmente, el indicador de efectividad del gobierno evalúa la fase final del proceso de responsividad, que es la de la producción e implementación de políticas públicas y leyes. La siguiente tabla expone estos indicadores y su fuente.

14. Gaceta Oficial de Bolivia: http://www.planificacion.gob.bo/uploads/normativa/A_DS214_123ESP.pdf. 


\section{TABLA III. DESCRIPCIÓN DE LOS INDICADORES}

\begin{tabular}{l|l|l}
\hline \multicolumn{1}{c|}{ INDICADOR } & \multicolumn{1}{|c}{ FUENTE } & \multicolumn{1}{c}{ PROXY } \\
\hline $\begin{array}{l}\text { Grado de } \\
\text { satisfacción con la } \\
\text { situación política } \\
\text { del país }\end{array}$ & Latinobarómetro & $\begin{array}{l}\text { Promedio anual por país para la pregunta «ंCómo } \\
\text { calificaría la situación política del país? ¿Diría Ud. que } \\
\text { es: Muy buena (1), buena (2), media (3), mala (4) o } \\
\text { muy mala (5)?». }\end{array}$ \\
\hline $\begin{array}{l}\text { El sistema partidista } \\
\text { y el electoral logran } \\
\text { procesar demandas }\end{array}$ & Latinobarómetro & $\begin{array}{l}\text { Puntuación para «¿cuánta confianza tiene Ud. en los } \\
\text { partidos políticos?» (\% respondiendo mucha o algo de } \\
\text { confianza). }\end{array}$ \\
\hline $\begin{array}{l}\text { Percepción de la } \\
\text { población sobre el } \\
\text { acceso a las arenas } \\
\text { decisorias }\end{array}$ & Latinobarómetro & $\begin{array}{l}\text { Promedio anual por país para «Algunas personas dicen } \\
\text { que la manera como uno vota puede hacer que las } \\
\text { cosas sean diferentes en el futuro. Otros dicen que } \\
\text { independientemente de cómo vote, no hará que las } \\
\text { cosas sean mejores en el futuro. ¿Cuál frase está más } \\
\text { cercana a su manera de pensar?». }\end{array}$ \\
\hline $\begin{array}{l}\text { Efectividad del } \\
\text { gobierno }\end{array}$ & $\begin{array}{l}\text { World } \\
\text { Governance } \\
\text { Indicators }\end{array}$ & $\begin{array}{l}\text { (Varía de aproximadamente -2.5 (débil) a 2,5 (fuerte) } \\
\text { en el desempeño de la gobernanza). Refleja la } \\
\text { percepción de la calidad de los servicios públicos, } \\
\text { la calidad de la administración pública y el grado de } \\
\text { independencia que tiene de las presiones políticas; } \\
\text { la calidad de la formulación e implementación } \\
\text { de políticas, y la credibilidad del compromiso del } \\
\text { gobierno con tales políticas. }\end{array}$ \\
\hline $\begin{array}{l}\text { Percepción sobre el } \\
\text { Legislativo }\end{array}$ & Latinobarómetro & $\begin{array}{l}\text { Porcentaje respondiendo «La democracia es preferible } \\
\text { arcentaje de personas que tienen alguna o mucha } \\
\text { confianza en el Congreso. }\end{array}$ \\
\hline
\end{tabular}

Fuente: Elaboración propia a partir de datos de opinión pública de encuestas del Latinobarómetro (http://www. latinobarometro.org/latino/latinobarometro.jsp) y Word Governance Indicators (http://info.worldbank.org/ governance/wgi/index.asp).

En seguida, estos indicadores fueron organizados en un panel que cubría el período de 1996 a 2010, según las encuestas encontradas para medir la opinión pública de Bolivia, Venezuela, Brasil y Chile. Para eso examinamos la variación entre países e intrapaíses porque consideramos la variación en el tiempo para aquellos que pasaron de gobiernos de derecha a gobiernos de izquierda. El método elegido para ejecutar las regresiones fue paneles con errores robustos corregidos, tal como proponen Beck y Katz ${ }^{15}$ (1995). Las dos variables:

15. De acuerdo con Beck y Katz (1995), las regresiones de paneles con errores robustos corregidos deben ser empleados para corregir problemas de heteroscedasticidad en los datos y de autocorrelación 
«Izquierda en Venezuela y Bolivia» e «Izquierda en Brasil y Chile» son dicotómicas, siendo 1 para gobiernos de izquierda y 0 para no gobiernos de izquierda. Es decir, el resultado es la comparación de los períodos en que la izquierda estuvo en el poder con los períodos en que la izquierda no estuvo. En este análisis, no se incluyeron variables de control por dos razones: primera, como son pocos casos, incluir muchas variables independientes podría perjudicar la adherencia de los datos, ya que el $\mathrm{N}$ es pequeño. Segunda, porque nuestro objetivo no es controlar el efecto de los gobiernos por otras variables, sino simplemente cuantificar el efecto, es decir, observar cómo el hecho de tener un gobierno de izquierda aumenta (o disminuye) las actitudes de la población hacia las dimensiones creadas.

Conforme a las informaciones de la Tabla IV los resultados varían tanto en lo que se refiere a la izquierda contestataria presente en Venezuela y Bolivia, cuanto a la izquierda moderada de Brasil y Chile.

En cuanto a los indicadores más generales sobre la adhesión a la democracia, los modelos 5 y 2 muestran que hubo un aumento considerable de los ciudadanos en Venezuela y Bolivia en el período analizado, presentando una puntuación del 13,6\%. El mismo indicador revela que cayó la adhesión de los ciudadanos gobernados por la izquierda moderada en Chile y Brasil, llegando a una puntuación del $-3,2 \%$. Pero, en contrapartida, en relación con la satisfacción con la situación política del país los coeficientes solamente son estadísticamente significativos para Brasil y Chile. Solo para estos países observamos la percepción de una mejora en la situación política cuando responden la siguiente pregunta: ¿Cómo describiría la situación política del país? Estos resultados parecen revelar que en cuanto régimen político la democracia sigue siendo bastante valorada por los ciudadanos de los países gobernados por la izquierda, pero entre esas naciones hay aún mayor adherencia en aquellos contextos administrados por la izquierda contestataria. Otro aspecto relevante de estos resultados es que la mejora en la situación política del país no es una condición suficiente para que haya mayor valorización de la democracia.

En lo que se refiere a los indicadores más directamente vinculados a la responsividad, usamos una cuestión que busca medir la eficiencia del voto en las democracias: «Algunas personas dicen que la manera como uno vota puede hacer que las cosas sean diferentes en el futuro. Otros dicen que independientemente de cómo vote, no hará que las cosas sean mejores en el futuro. ¿Cuál frase está más cercana a su manera de pensar?». Aquí, asumimos que aquellos ciudadanos que consideran su voto eficiente están implícitamente reconociendo que los políticos son sensibles a la voluntad popular expresada en las urnas. Como se puede ver en el modelo 3 de la tabla, solo en Brasil y en Chile ese indicador tuvo un efecto positivo, lo que puede ser explicado por el nivel más elevado del proceso de institucionalización electoral y por la presencia de instituciones democráticas estables, mostrando así una mayor adhesión de la opinión pública de esos países al instituto del voto.

temporal. Se calcularon utilizando Stata por medio del comando «xtpcse», y comparado con MQOs porque los coeficientes son más consistentes. 
TABLA IV. INDICADORES DE RESPONSIVIDAD

\begin{tabular}{l|c|c|c|c|c|c}
\hline & $(1)$ & $(2)$ & $(3)$ & $(4)$ & $(5)$ & $(6)$ \\
\hline & $\begin{array}{c}\text { Percepción } \\
\text { sobre la } \\
\text { efectividad } \\
\text { del gobierno }\end{array}$ & $\begin{array}{c}\text { Grado de } \\
\text { satisfacción con } \\
\text { la situación } \\
\text { política del país }\end{array}$ & $\begin{array}{c}\text { Percepción } \\
\text { sobre la } \\
\text { efectividad } \\
\text { del voto }\end{array}$ & $\begin{array}{c}\text { Confianza } \\
\text { en los } \\
\text { partidos } \\
\text { politicos }\end{array}$ & $\begin{array}{c}\text { Percepción } \\
\text { sobre la } \\
\text { democracia }\end{array}$ & $\begin{array}{c}\text { Confianza } \\
\text { en el } \\
\text { Congreso }\end{array}$ \\
\hline $\begin{array}{l}\text { Izquierda en } \\
\text { Venezuela y } \\
\text { Bolivia }\end{array}$ & $-0.596^{* * *}$ & -0.0260 & 2.916 & 1.908 & $13.55^{* * *}$ & 2.303 \\
\cline { 2 - 7 } & $(-18.25)$ & $(-0.49)$ & $(0.87)$ & $(1.35)$ & $(5.36)$ & $(1.73)$ \\
\hline $\begin{array}{l}\text { Izquierda en } \\
\text { Chile y Brasil }\end{array}$ & $0.672^{* * *}$ & $-0.0940^{*}$ & $5.901^{* * *}$ & $1.842^{*}$ & $-3.150^{*}$ & $7.209^{* * *}$ \\
\cline { 2 - 7 } & $(21.61)$ & $(-2.30)$ & $(4.88)$ & $(2.18)$ & $(-2.13)$ & $(7.39)$ \\
\hline \multirow{2}{*}{ Constante } & $-0.101 * * *$ & $3.370^{* * *}$ & $54.32^{* * *}$ & $18.64 * * *$ & $54.31 * * *$ & $23.63 * * *$ \\
\cline { 2 - 7 } & $(-3.97)$ & $(38.33)$ & $(34.94)$ & $(16.24)$ & $(40.28)$ & $(18.73)$ \\
\hline Observaciones & 84 & 35 & 56 & 98 & 98 & 70 \\
\hline
\end{tabular}

Nota: Estadísticas t entre paréntesis * $\mathrm{p}<0.05$; ** $\mathrm{p}<0.01$; *** $\mathrm{p}<0.001$.

Fuente: Elaboración propia.

La variable tiempo de existencia de esas instituciones es fundamental en este sentido, ya que tanto en Bolivia como en Venezuela la desconfianza de la población en las instituciones electorales y partidarias tradicionales resultó en la quiebra de ese sistema de representación. En ese contexto de inestabilidad institucional se reorganizó el sistema político actual, en el cual hubo inserción de nuevos actores sociales y otras formas de participación directa en los procesos decisorios de aquellas sociedades. Aspecto reforzado por otro indicador crucial en la fase de estructuración de las preferencias electorales, la confianza en los partidos políticos, considerada en el modelo 6. La percepción de confianza en los partidos políticos solo mejoró en Brasil y Chile, pero no en Venezuela y Bolivia, donde los principales partidos que están gobernando estos países se organizaron recientemente. Así como por el indicador que mide la confianza en el Congreso Nacional, que como podemos comprobar también solo aumentó y fue estadísticamente positivo en el período analizado en los países gobernados por la izquierda moderada, llegando la puntuación al 7,2\% más que en los países gobernados por la derecha.

Finalmente, el último indicador -el modelo 1- refleja la percepción de los ciudadanos sobre la calidad de los servicios públicos, la calidad de la administración pública, la calidad de la formulación y aplicación de políticas y la credibilidad del compromiso del gobierno con esas políticas. Buscamos, así, evaluar la última fase del proceso de responsividad de un gobierno, que es la etapa de la implementación de las políticas públicas y de las leyes. En este aspecto, los gobiernos de Brasil y Chile muestran coeficientes positivos mientras que en Venezuela y Bolivia los coeficientes son negativos. Este resultado es interesante porque muestra que mientras los gobiernos de izquierda moderada mejoraron en la evaluación de 
los ciudadanos sobre la efectividad del gobierno, en las izquierdas contestatarias este aspecto empeoró, lo que refuerza la idea de que es necesario clasificar a las izquierdas en dos subtipos, pues hay diferencias importantes en la percepción de los ciudadanos de cada ambiente conforme al desempeño del grupo político en administrar e implementar las demandas del electorado. Por los resultados observados en la Tabla IV la izquierda moderada ha logrado ser más responsiva a esos anhelos de la población.

En general, las evidencias examinadas revelan que existen diferencias significativas en cuanto al grado de responsividad, según la percepción del ciudadano, entre los dos subtipos de izquierda. Aunque es un análisis exploratorio, y para profundizarla tendremos que insertar otras variables en los modelos, es posible concluir que la izquierda moderada en Chile y Brasil viene alcanzando mayor grado de responsividad en las tres etapas presentadas por Powell: de la estructuración de las preferencias electorales con los indicadores 3 y 4, de la formación de los gobiernos con el indicador 6 y de la producción de las políticas y las leyes con los indicadores 1 y 2 (Tabla IV).

\section{CONSIDERACIONES FINALES}

La literatura sobre el giro a la izquierda en América Latina nos mostró que hay distintos gobiernos de izquierda en la región. En el límite, una izquierda moderada (como los gobiernos de Lula en Brasil y de Bachelet en Chile) y una izquierda contestataria (con los gobiernos de Chávez en Venezuela y Evo Morales en Bolivia). Ante ese cuadro político y social de esa región, nos preguntamos si esos distintos gobiernos de izquierda ejercieron impactos diferenciados sobre la calidad de la democracia, más específicamente, en lo que se refiere a una de sus dimensiones: la responsividad. Es decir, ¿los distintos gobiernos de izquierda presentaron distintos grados de responsividad? Esta fue la cuestión central que buscamos responder en este texto. Para ello, evaluamos esa dimensión elaborando indicadores a partir de la percepción de los ciudadanos en el período de 1996 a 2014.

El análisis realizado aquí demostró que hay diferencias en el grado de responsividad entre gobiernos de izquierda, así como apunta al hecho de que los diferentes tipos de izquierda (moderada y contestataria) presentaron distintos impactos sobre el grado de responsividad. La izquierda moderada reveló, en la suma de los indicadores, mayores grados de responsividad comparada a los gobiernos de la izquierda contestataria.

$\mathrm{Al}$ contraponer los datos calculados a partir de los indicadores de responsividad con los aspectos políticos institucionales y los cambios emprendidos por los distintos gobiernos de izquierda, confirmamos las diferencias de los gobiernos. Notamos que, en lo que se refiere a los aspectos institucionales, la izquierda contestataria de Bolivia y Venezuela emprendió cambios fundamentales en el ambiente institucional, con la inserción de mecanismos de participación e inclusión, inscritos en las nuevas constituciones promulgadas por estos gobiernos. Así, con mecanismos más inclusivos y con la inserción de innumerables canales de participación los gobiernos de la izquierda contestataria parecerían más responsivos que los de la izquierda moderada en lo que se refiere a la incorporación de mayores y diversos segmentos de la población en el sistema de representación. Sin embargo, el análisis del grado de responsividad a partir de la percepción de los ciudadanos demostró exactamente lo contrario, 
o sea, la incorporación de esos mecanismos más cercanos a la democracia directa no garantizó la percepción de la mejora con respecto a sus instituciones.

En este sentido, podemos concluir que lo que explica este resultado es que la izquierda moderada (Chile y Brasil) se encuentra en un contexto institucionalizado con un sistema de partidos mejor estructurado, lo que facilita la confianza de los ciudadanos en las instituciones políticas. En el caso de Bolivia y Venezuela, la izquierda contestataria (Evo Morales y Chávez) asume el poder en un contexto en que las instituciones políticas están deslegitimadas. En Bolivia, además, los movimientos sociales representan mucho más los canales de expresión de la voluntad popular que los partidos. En Venezuela, la dependencia del partido oficialista de la figura de Chávez y la inhabilidad de los partidos de oposición crearon un escenario de conflicto dificultando la institucionalización del sistema partidista, dejando un escenario de inseguridad en cuanto al futuro del país.

En suma, la izquierda moderada situada en contextos de mayor estabilidad política-electoral tuvo un efecto positivo en cuanto al grado de responsividad. La izquierda contestataria situada en procesos de quiebra de hegemonía y refundación del Estado, con la presencia de nuevos actores, aún no ha logrado completar el proceso de institucionalización y, por lo tanto, presenta grados de responsividad menores, en comparación con los de la izquierda moderada.

\section{BIBLIOGRAFÍA}

Alcántara Sáez, M. (2008, septiembre-octubre). La escala de la izquierda. La ubicación ideológica de presidentes y partidos de izquierda en América Latina. Nueva Sociedad, 217. https://nuso.org/ articulo/la-ubicacion-ideologica-de-presidentes-y-partidos-de-izquierda-en-america-latina/.

Alcántara Sáez, M. (2009, julio). Los partidos y la política en América Latina al inicio de 2009. Revista Latinoamericana de Politica Comparada. CELAEP, 2, 71-83.

Altman, D. y Pérez-Liñan, A. (2002). Assessing the Quality of Democracy: Freedom, Competitiveness and Participation in Eighteen Latin American Countries. Democratization, 9(2), 85-100. https://doi. org/10.1080/714000256.

Asamblea Nacional Constituyente. (1999). Constitución de la República Bolivariana de Venezuela. Caracas: MINCI.

Batista, P. N. (1994). O Consenso de Washington. A visão neoliberal dos problemas latino-americanos. En B. Lima Sobrinho (Ed.), Em defesa do interesse nacional: desinformação e alienação do patrimônio público (pp. 99-144). Rio de Janeiro: Paz e Terra.

Beck, N. y Katz, J. N. (1995). What to Do (and Not To Do) with Time Series Cross-Section Data. American Political Science Review, 89, 634-647. https://doi.org/10.2307/2082979.

Bobbio, N. (1995). Direita e esquerda: raz̃ões e significados de uma distinção politica. São Paulo: Editora UNESP.

Dahl, R. (1971). Polyarchy: Participation and Opposition. New Haven: Yale Univ. Press.

Diamond, L. y Morlino, L. (Eds.) (2005). Assessing the Quality of Democracy. Baltimore: Johns Hopkins Univ. Press.

Domínguez, J. y Shifter, M. (2008). Constructing Democratic Governance in Latin America (3 ${ }^{\text {rd }}$ ed.). Baltimore: Johns Hopkins Univ. Press.

Freidenberg, F. y Caminotti, M. (2018). Reformas electorales inclusivas en América Latina y retos para la igualdad real de las mujeres en la política. En F. Freidenberg, M. Caminotti, B. Muñoz-Pogossian y T. Došek (Eds.), Mujeres en la Política. Experiencias nacionales y subnacionales en América Latina (pp. 7-32). Ciudad de Mexico: IECM-UNAM-IFE. 
Freidenberg, F. y Došek, T. (2016). Las reformas electorales en América Latina (1978-2015). En K. Casas-Zamora, R. Chanto, B. Muñoz-Pogossian y M. Vidaurri (Eds.), Las reformas políticas en América Latina: Tendencias y casos (pp. 25-92). Washington D.C.: Organización de los Estados Americanos.

Hagopian, F. (2005). Chile and Brazil. En L. Diamond y L. Morlino, Assessing the Quality of Democracy. Baltimore: Johns Hopkins Univ. Press.

Hagopian, F. y Mainwaring, S. (Eds.) (2005). The third wave of democratization in Latin America: Advances and Setbacks. Cambridge: Cambridge Univ. Press.

Harvey, D. (2007). Breve historia del neoliberalismo. Madrid: Akal.

Levine, D. H. y Molina, J. E. (2007). La calidad de la democracia en América Latina: una visión comparada. América Latina Hoy, 45, 17-46. http://dx.doi.org/10.14201/alh.2427.

Levine, D. y Molina, J. E. (Eds.) (2011). The Quality of Democracy in Latin America. Boulder: Lynne Rienner.

Londoño, J. F. y Zovatto, D. (2015). Capítulo 5. América Latina. En E. Falguera, S. Jones y M. Ohman (Eds.), Financiamento de partidos politicos e campanbas eleitorais: um manual sobre financiamento politico (pp. 181-232). Rio de Janeiro: FGV Editora.

Madrid, R. (2008). Leftist Government in Latin America: A Comparison of Bolivia, Brazil, Chile, and Venezuela. LLilas Portal Issue, 3, 14-17. http://hdl.handle.net/2152/61880.

Madrid, R. L., Hunter, W. y Weyland, K. (2010). The Policies and Performance of the Contestatory and Moderate Left. En K. Weyland, R. Madrid y W. Hunter, Leftists Governments in Latin América: Successes and Shortcomings (pp. 140-180). Cambridge: Cambridge University Press. https://doi. org/10.1017/CBO9780511778742.007.

Mainwaring, S. y Scully, T. (Eds.) (2010). Democratic Governance in Latin America. Stanford: Stanford Univ. Press.

Mainwaring, S. y Torcal, M. (2005). La institucionalización de los sistemas de partidos y la teoría del sistema partidista después de la tercera ola democratizadora. América Latina Hoy, 41, 141-173. http://dx.doi.org/10.14201/alh.2442.

Melo, C. R. (2006). Reforma Política em Perspectiva Comparada na América do Sul. En L. Avritzer y F. Anastasia, Reforma politica no Brasil (pp. 45-62). Belo Horizonte: Editora UFMG.

Negretto, G. L. (2009). La reforma electoral en América Latina: Entre el interés partidario y las demandas ciudadanas. En A. Fontaine et al., Reforma del Sistema Electoral Chileno (pp. 63-104). Santiago: PNUD, CEP, Libertad y Desarrollo, Proyectamérica y Cieplan.

Nicolau, J. (2004). Sistemas eleitorais. Rio de Janeiro: Editora FGV.

Ocampo, J. A. y Ros, J. (2011). Shifting paradigms in Latin America's economic development. En J. A. Ocampo y J. Ros (Eds.), The Oxford Handbook of Latin American Economics. Oxford: Oxford University Press. doi: 10.1093/oxfordhb/9780199571048.001.0001.

Payne, M., Zovatto, D. y Mateo Díaz, M. (Eds.) (2006). La política importa: democracia y desarrollo en América Latina. Banco Interamericano de Desarrollo.

Pinto, Á. y Flisfisch, A. (2011). El Estado de Ciudadanía: transformaciones, logros y desafíos del Estado en América Latina en el siglo XXI (1. ${ }^{\mathrm{a}}$ ed.). Buenos Aires: Sudamericana.

Pogrebinschi, T. (2013). El giro pragmático de la democracia en América Latina. Nueva Sociedad, 1(24), 1-25, Argentina: Fundación Friedrich Ebert. http://library.fes.de/pdf-files/nuso/10084.pdf.

Powell, B. (2005). The Chain of Responsiveness. En L. Diamond y L. Morlino (Eds.), Assessing the Quality of Democracy. Baltimore: Johns Hopkins Univ. Press.

Roberts, K. M. (2008). ¿Es posible una socialdemocracia en América Latina? Nueva Sociedad, 217, 86-98. https://www.nuso.org/media/articles/downloads/3549_1.pdf.

Varas, A. (2012). La democracia frente al poder. Chile 1990-2010. Santiago, Chile: Catalonia. 
MARIA DO SOCORRO SOUSA BRAGA Y KÁTIA ALVES FUKUSHIMA

LA CALIDAD DE LA RESPONSIVIDAD DE GOBIERNOS DE IZQUIERDA EN PAÍSES DE AMÉRICA LATINA: LA PERCEPCIÓN DE LA CIUDADANÍA

Weyland, K. (2000). Os riscos na reestruturação econômica da América Latina: lições da teoria prospectiva. Opinião Pública, 6(1), 76-108. http://dx.doi.org/10.1590/S0104-62762000000100004.

Weyland, K. (2009). The rise of Latin America's two lefts: Insights from rentier state theory. Comparative Politics, 41(2), 145-164. https://doi.org/10.5129/001041509X12911362971918. 
